\title{
Queens on Non-square Tori
}

\author{
Grant Cairns \\ Department of Mathematics \\ La Trobe University, Melbourne, Australia 3086 \\ G.Cairns@latrobe.edu . au
}

Submitted: January 29, 2001; Accepted: June 11, 2001.

MR Subject Classifications: 05C69, 05B99

\begin{abstract}
We prove that for $m<n$, the maximum number of nonattacking queens that can be placed on the $n \times m$ rectangular toroidal chessboard is $\operatorname{gcd}(m, n)$, except in the case $m=3, n=6$.
\end{abstract}

The classical $n$-queens problem is to place $n$ queens on the $n \times n$ chessboard such that no pair is attacking each other. Solutions for this problem exist for all for $n \neq 2,3$ [1]. The queens problem on a rectangular board is of little interest; on the $n \times m$ board for $m<n$, one can obviously place at most $m$ nonattacking queens and for $4 \leq m<n$, one can just take a solution on the $m \times m$ board and extend the board. Moreover, the reader will easily find solutions on the $3 \times 2$ and $4 \times 3$ boards and so these give solutions on the $n \times 2$ and $n \times 3$ boards for all $3 \leq n$ and $4 \leq n$ respectively.

In chess on a torus, one identifies the left and right edges and the top and bottom edges of the board. On the $n \times n$ toroidal board, the $n$-queens problem has solutions when $n$ is not divisible by 2 or 3 [3], and the problem of placing the maximum number of queens when $n$ is divisible by 2 or 3 is completely solved in [2]. The traditional $n$-queens problem and the toroidal $n$-queens problem are closely related, both logically and historically (see [4]). However, unlike the rectangular traditional board, the queens problem on the rectangular toroidal board is interesting and non-trivial and yet it seems that it has not been studied.

In order to work on the toroidal board we use the ring $\mathbb{Z}_{i}=\mathbb{Z} /(i)$, which we identify with $\{0, \ldots, i-1\}$, and the natural ring epimorphism $\mathbb{Z} \rightarrow \mathbb{Z}_{i} ; x \mapsto[x]_{i}$, where $[x]_{i}$ is to be interpreted as the remainder of $x$ on division by $i$. We give the squares of the $n \times m$ toroidal board coordinate labels $(x, y), x \in \mathbb{Z}_{m}, y \in \mathbb{Z}_{n}$, in the obvious way. The positive (resp. negative) diagonal is the subgroup $P=\left\{\left([x]_{m},[x]_{n}\right) ; x \in \mathbb{Z}\right\}$ (resp. $\left.N=\left\{\left([x]_{m},[-x]_{n}\right) ; x \in \mathbb{Z}\right\}\right)$. Notice that the diagonals are both subgroups of $\mathbb{Z}_{m} \times \mathbb{Z}_{n}$ of index $\operatorname{gcd}(m, n)$. In addition, there is the vertical subgroup $V=\left\{\left(0,[x]_{n}\right) ; x \in \mathbb{Z}\right\}$ which has index $m$, and the horizontal subgroup $H=\left\{\left([x]_{m}, 0\right) ; x \in \mathbb{Z}\right\}$ which has index $n$. Queens at distinct positions $\left(x_{1}, y_{1}\right),\left(x_{2}, y_{2}\right)$ are nonattacking if and only if $\left(x_{1}, y_{1}\right)$ 
and $\left(x_{2}, y_{2}\right)$ belong to distinct cosets of $V, H, P$ and $N$. In particular, the $n \times m$ toroidal board can support no more than $\operatorname{gcd}(m, n)$ nonattacking queens.

The aim of this paper is to prove the

Theorem. For $m<n$, the maximum number of nonattacking queens that can be placed on the $n \times m$ rectangular toroidal chessboard is $\operatorname{gcd}(m, n)$, except in the case $m=3, n=6$.

Proof. First let $d=\operatorname{gcd}(m, n)$ and suppose that $d \neq 3$. Notice that in order to place $d$ nonattacking queens on the $n \times m$ toroidal board, it suffices to place $d$ nonattacking queens on the $2 d \times d$ toroidal board. Indeed, although the natural injection $\mathbb{Z}_{d} \times \mathbb{Z}_{2 d} \hookrightarrow \mathbb{Z}_{m} \times \mathbb{Z}_{n}$ is not in general a group homomorphism, it is easy to see that if two queens are nonattacking in $\mathbb{Z}_{d} \times \mathbb{Z}_{2 d}$, their images in $\mathbb{Z}_{m} \times \mathbb{Z}_{n}$ are also nonattacking. Thus, without loss of generality, we may assume that $n=2 m$. In this case $\operatorname{gcd}(m, n)=m$.

If $m \equiv 1,2,4,5(\bmod 6)$, a solution is easily obtained by placing a queen at each point in the set $A=\left\{(i, 2 i) ; i \in \mathbb{Z}_{m}\right\}$. Indeed, it is clear that no two distinct elements of $A$ belong to the same coset of $H$ or $V$. If elements $(i, 2 i)$ and $(j, 2 j)$ belong to the same coset of $P$, then $i-j \equiv 2 i-2 j(\bmod m)$ and so $i \equiv j(\bmod m)$ which implies $i=j$. If elements $(i, 2 i)$ and $(j, 2 j)$ belong to the same coset of $N$, then one has $3 i \equiv 3 j(\bmod m)$ which also gives $i=j$ when $m$ is not divisible by 3 .

Now suppose that $m$ is divisible by 6 , say $m=2^{k} .6 . l$, where $l$ is odd. Here the situation is slightly more complicated; a solution is obtained by placing queens at positions $(i, f(i))$, for $i=0, \ldots, m-1$, where

$$
f(i)= \begin{cases}2 i+[i]_{6 l} & ; \text { if }[i]_{3 l}=[i]_{6 l}, \\ 2 i+1+[i]_{6 l} & ; \text { otherwise }\end{cases}
$$

The case where $m \equiv 3(\bmod 6)$ is a good deal more complicated; we consider two subcases. First if $m \equiv 3(\bmod 12)$, say $m=12 k+3$, a solution is obtained by placing queens at positions $(i, g(i))$, for $i=0, \ldots, m-1$, where

$$
g(i)= \begin{cases}3 i & ; \text { if } i \leq 4 k \\ 2 & ; \text { if } i=4 k+1 \\ 2+m & ; \text { if } i=4 k+3 \\ 3 i-m+4 & ; \text { if } 4 k+2 \leq i \leq 10 k \text { and } i \text { is even, } \\ 3 i-m+2 & ; \text { if } i=10 k+2 \\ 3 i-m-4 & ; \text { if } 4 k+5 \leq i \leq 10 k+3 \text { and } i \text { is odd } \\ 3 i-m & ; \text { if } i \geq 10 k+4 .\end{cases}
$$

On the other hand, if $m \equiv 9(\bmod 12)$, say $m=12 k+9$, a solution is obtained by placing 
queens at positions $(i, h(i))$, for $i=0, \ldots, m-1$, where

$$
h(i)= \begin{cases}3 i & ; \text { if } i \leq 4 k+2 \\ 2 & ; \text { if } i=4 k+3 \\ 2+m & ; \text { if } i=4 k+5 \\ 3 i-m+4 & ; \text { if } 4 k+4 \leq i \leq 10 k+6 \text { and } i \text { is even, } \\ 3 i-2 m-2 & ; \text { if } i=10 k+8 \\ 3 i-m-4 & ; \text { if } 4 k+7 \leq i \leq 10 k+7 \text { and } i \text { is odd } \\ 3 i-m & ; \text { if } i \geq 10 k+9 .\end{cases}
$$

The verification that the above functions $f, g$ and $h$ have the required properties is tedious but elementary.

It remains to deal with the case where $\operatorname{gcd}(m, n)=3$. Here the reader will readily find that there is no solution on the $6 \times 3$ board, but there are solutions on the $9 \times 3$ board. It follows that there are solutions on the $n \times m$ board for all $m<n$ with $\operatorname{gcd}(m, n)=3$ except in the case $m=3, n=6$. This completes the proof of the theorem.

\section{References}

[1] W. Ahrens, Mathematische Unterhaltungen und Spiele, B.G. Teubner, 1921.

[2] P. Monsky, Problem E3162, Amer. Math. Monthly 96 (1989), 258-259.

[3] G. Pólya, Über die "doppelt-periodischen" Lösungen des n-Damen-Problems, George Pólya: Collected papers Vol. IV (G.-C. Rota, ed.), pages 237-247, MIT Press, Cambridge, London, 1984.

[4] I. Rivin, I. Vardi and P. Zimmermann, The $n$-queens problem, Amer. Math. Monthly 101 (1994), 629-639. 